\title{
Nitrogen losses from the human small bowel: obligatory losses and the effect of physical form of food
}

\author{
A CHACKO AND J H CUMMINGS \\ From the MRC Dunn Clinical Nutrition Centre; Cambridge, and Wellcome Research Unit, Christian Medical \\ College, Vellore, India
}

SUMmaRY The amount and form of nitrogen lost from the human small intestine and the dietary factors which influence it have been studied in six ileostomists. Over a six day period the subjects were fed a series of diets including low nitrogen (LND) $0 \cdot 17 \mathrm{~g} \mathrm{~N} / \mathrm{day}, \mathrm{LND}+$ soya beans $(5 \cdot 87 \mathrm{~g} \mathrm{~N} / \mathrm{day})$ and a high fibre diet (HFD) (10.6 g N/day). The soya beans were fed either whole or pureed to test the effect of physical form of food. Total $N$, protein, amino acids, urea, and ammonia were measured in ileostomy effluent which was collected throughout the study. Total $N$ excretion was LND 0.91 (0.04) (SE) g/day; LND + whole soya beans (WSB) $2 \cdot 26(0 \cdot 15)$ g/day; LND + pureed soya beans (PSB) 1.42 $(0 \cdot 12)$ g/day (WSB $v$ PSB, p $<0 \cdot 001)$; and HFD $2 \cdot 17(0 \cdot 11)$ g/day (HFD $v$ PSB, $<<0 \cdot 001$, HFD $v$ WSB, $\mathrm{NS})$. $\mathrm{N}$ losses as urea, ammonia, and free amino acids were less than $10-15 \%$ of total $\mathrm{N}$, the remainder being protein (48-51\%) and (by difference) peptides (20-30\%). Eighty to $85 \%$ of effluent $\mathbf{N}$ was in the insoluble (pellet) fraction except on the low $\mathbf{N}$ diet where it was $66 \%$. The physical form. of food clearly influenced $\mathrm{N}$ digestibility in the soya beans whilst changes in dietary fibre seem not to have a significant effect.

The human large intestine plays a significant role in the digestion of dietary carbohydrates especially starch and non-starch polysaccharides (dietary fibre).' It has only recently been appreciated that significant protein breakdown may also occur in the colon. ${ }^{2}$ Total nitrogen reaching the human large bowel from the terminal ileum is in the range $0 \cdot 5-4 \cdot 0$ $\mathrm{g} /$ day. ${ }^{3-6}$ It is assumed that this is a mixture of endogenous nitrogen and dietary residues. The relative contributions from the two sources, and the form in which they enter the large bowel are not known. There are also few data available on the influence of dietary factors on small bowel nitrogen losses. Gibson $e t a l^{6}$ have shown that increasing dietary protein from $40-100 \mathrm{~g} /$ day increased mean daily nitrogen losses in ileostomy effluent from $1 \cdot 8-2 \cdot 6 \mathrm{~g} /$ day. In another study with ileostomists Sandberg et al ${ }^{7}$ showed that supplementing a low fibre diet with $16 \mathrm{~g}$ wheat bran does not change nitrogen loss.

Nitrogen entering the large intestine may exert a significant effect on colonic function particularly production of ammonia, phenolic compounds and

Address for correspondence: Dr J H Cummings, MRC Dunn (linical Nutrition Centre, 100 Tennis Court Road, Cambridge CB2 IOI.

Received for publication 16 December 1987 amines. ${ }^{89}$ Because the factors controlling nitrogen loss from the small intestine are largely unknown we have, using ileostomy subjects as a model, attempted to (1) measure endogenous nitrogen losses, (2) determine the forms of nitrogen loss and the effect of diet on the different fractions, (3) test the effect of change in physical form of food, and (4) assess the role of dietary fibre.

\section{Methods}

PATIENTS

Six healthy ileostomists aged 38-66 years (mean 53 years) were studied. All had total proctocolectomy with terminal ileostomy carried out for ulcerative colitis at least two years before the study. All subjects had less than $5 \mathrm{~cm}$ terminal ileum resected. They had normal dentition and were otherwise healthy with normally functioning ileostomies.

PROTOCOL

The study lasted six days during which time the subjects lived in a metabolic suite at the Dunn Clinical Nutrition Centre. On the day preceding the start of the study all subjects ate a low nitrogen diet 
from $5 \mathrm{pm}$ onwards. On days 1 and 2 , the low nitrogen diet (LND) was continued, after which three further diets were given in random order. These diets were LND+whole soya beans (WSB) one day, LND+pureed soya beans (PSB) one day, and high fibre diet (HFD) two days. Throughout the study ileostomy output was collected every two hours from 9 am to $9 \mathrm{pm}$. The 12 hour overnight collection was pooled as a single sample. Samples of ileostomy discharge were collected directly from the stoma from all subjects for urea and ammonia estimation and microbial counts. Immediately after collection the sample was weighed and homogenised in a stomacher (Lab Blender 400, Seward Laboratory, London, UK) for four minutes. Aliquots were taken and stored at $-20^{\circ} \mathrm{C}$. A weighed aliquot was centrifuged at $3000 \mathrm{~g}$ for $30 \mathrm{~min}$. The supernatant (soluble fraction) and pellet (insoluble fraction) were separated and stored at $-20^{\circ} \mathrm{C}$. The bacteria in the sample for urea and ammonia estimation were inactivated with absolute ethanol " (three parts fluid to one part ethanol) and stored at $-20^{\circ} \mathrm{C}$. The samples for microbial counts were collected in sterile containers and serially diluted in half strength nutrient broth. Aliquots $(0 \cdot 1 \mathrm{ml})$ were then plated on either WilkinsChalgren anaerobe agar (OXOID - Code CM 619) or nutrient agar (OXOID - Code CM 3/4) plates. The Wilkins-Chalgren plates had been preincubated for $48 \mathrm{~h}$ in an anaerobic cabinet $\left(80 \% \mathrm{~N}_{2}, 10 \% \mathrm{CO}_{2}\right.$, $10 \% \mathrm{H}_{2}$ atmosphere) at $37^{\circ} \mathrm{C}$ for up to five days. The nutrient agar plates were incubated anaerobically at $37^{\circ} \mathrm{C}$ for $48 \mathrm{~h}$. Breath hydrogen was measured daily in all subjects using an electrochemical detector (Exhaled hydrogen monitor, GMI, Scotland) in the fasting state and two to three hours postprandially.

DIETS

All diets were prepared in the metabolic kitchen. The subjects did not eat any food other than that given from the kitchen. Any food left was weighed and recorded. Duplicates of each meal were homogenised and pooled aliquots frozen and stored at $-20^{\circ} \mathrm{C}$ for nitrogen estimation. Details of the diet are given in Tables 1 and 2. Diet I was LND which provided $0.17 \mathrm{~g} \mathrm{~N} /$ day. It was used to measure endogenous nitrogen losses. Diet II had $100 \mathrm{~g}$ whole soya beans boiled at $100^{\circ} \mathrm{C}$ for one hour and given in the form of a salad in addition to LND. The subjects ate part of the soya beans with breakfast and the rest with lunch. Diet III was similar to Diet II except that the soya beans were ground in an electric blender before cooking (pureed soya beans). Diet IV was designed to contain about twice the average national fibre intake for the UK. The fibre was contributed by wholemeal bread, breakfast cereals, fruits and vegetables.
Table 1 Diets

\begin{tabular}{|c|c|c|c|c|}
\hline & Low nitrogen diet (Diet I) & & High fibre diet (Diet IV) & \\
\hline \multirow[t]{5}{*}{$9 \mathrm{am}$} & Low protcin bread & $40 \mathrm{~g}$ & Wectabix & $40 \mathrm{~g}$ \\
\hline & (toasted) & & Wholemeal bread & $50 \mathrm{~g}$ \\
\hline & Margarine & $10 \mathrm{~g}$ & Butter/Flora & $50 \mathrm{~g}$ \\
\hline & Marmalade & $20 \mathrm{~g}$ & Milk & $2(x) \mathrm{g}$ \\
\hline & & & Marmalade & $15 \mathrm{~g}$ \\
\hline $11 \mathrm{am}$ & Hycal ice cream & $50 \mathrm{~g}$ & Flapjack & 6) $g$ \\
\hline \multirow[t]{5}{*}{$1 \mathrm{pm}$} & Low protein bread & $40 \mathrm{~g}$ & Wholemeal bread & $100 \mathrm{~g}$ \\
\hline & Tomato & $30 \mathrm{~g}$ & Chicken & $40 \mathrm{~g}$ \\
\hline & Margarine & $10 \mathrm{~g}$ & Tomato & $50 \mathrm{~g}$ \\
\hline & Hycal ice cream & $50 \mathrm{~g}$ & Butter/Flora & $20 \mathrm{~g}$ \\
\hline & & & Pear & $100 \mathrm{~g}$ \\
\hline \multirow[t]{3}{*}{$3 \mathrm{pm}$} & Pear & $50 \mathrm{~g}$ & Banana & $100) \mathrm{g}$ \\
\hline & Hycal ice cream & $50 \mathrm{~g}$ & Wholemeal bread & $25 \mathrm{~g}$ \\
\hline & & & Butter/Flora & $5 \mathrm{~g}$ \\
\hline \multirow[t]{5}{*}{$5 \mathrm{pm}$} & Low protcin bread & $40 \mathrm{~g}$ & Beef & $50 \mathrm{~g}$ \\
\hline & Margarine & $10 \mathrm{~g}$ & Carrots & $80 \mathrm{~g}$ \\
\hline & Jam & $20 \mathrm{~g}$ & Potatoes & $150 \mathrm{~g}$ \\
\hline & Hycal ice cream & $50 \mathrm{~g}$ & Gravy & 60) $\mathrm{g}$ \\
\hline & & & Apricot crumble & $110 \mathrm{~g}$ \\
\hline \multirow[t]{5}{*}{$7 \mathrm{pm}$} & Hycal ice cream & $50 \mathrm{~g}$ & Wholemcal bread & $25 \mathrm{~g}$ \\
\hline & & & Butter/Flora & $5 \mathrm{~g}$ \\
\hline & Sweets (daily) & $43 \mathrm{~g}$ & & \\
\hline & Lemon juice (daily) & $5 \mathrm{~g}$ & & \\
\hline & $\begin{array}{l}\text { Black tea and coffee as } \\
\text { wished }\end{array}$ & & $\begin{array}{l}\text { Black tea and coffec } \\
\text { as wished }\end{array}$ & \\
\hline
\end{tabular}

On Diet II patients ate soya bean salad in addition to Low Nitrogen Diet. Ingredients of soya bean salad were as follows: Whole soya beans $100 \mathrm{~g}$, tomato $50 \mathrm{~g}$. French dressing $20 \mathrm{ml}$. Part of the salad was eaten at 9 am and the rest at $1 \mathrm{pm}$. Diet III was similar to Diet II except that the soya beans were pureed.

\section{CHEMICAL METHODS}

Nitrogen was measured in all samples of effluent homogenate and in soluble and insoluble fractions by the Dumas method" (Carlo Erba, Milan ANA 1500 nitrogen analyser). To estimate protein, the sample was first precipitated with $10 \%(\mathrm{w} / \mathrm{v})$ Trichloroacetic acid (TCA) and centrifuged at $15000 \mathrm{~g}$ for $10 \mathrm{~min}$. The pellets were solubilised and resuspended in $0 \cdot 1 \mathrm{M} \mathrm{NaOH}$. Proteins were then determined by the Lowry method. ${ }^{2}$ To determine free amino acids samples from three subjects were precipitated with $4 \%$ sulphosalicylic acid and centrifuged at $3000 \mathrm{~g}$ for

Table 2 Composition of diets*

\begin{tabular}{|c|c|c|c|}
\hline & $\begin{array}{l}\text { Low N diet } \\
\text { (Diet I) }\end{array}$ & $\begin{array}{l}\text { Low } N \text { diet }+ \text { soya beans } \\
\text { (Diets IIIIII) }\end{array}$ & $\begin{array}{l}\text { High fibre diet } \\
\text { (Diet IV) }\end{array}$ \\
\hline Energy (Kcals) & 1618 & 2157 & 2042 \\
\hline MJ & $6 \cdot 8$ & $9 \cdot 02$ & $8 \cdot 6$ \\
\hline Nitrogen & $0 \cdot 17$ & $5 \cdot 87$ & $10 \cdot 6$ \\
\hline Protein & $1 \cdot() 6$ & $35 \cdot 6$ & $65 \cdot 2$ \\
\hline Fat & $68 \cdot 8$ & $101 \cdot 1$ & $81 \cdot 3$ \\
\hline Carbohydrate g & $258 \cdot 1$ & $278 \cdot 1$ & $281 \cdot 7$ \\
\hline Fibre & 1.94 & $15 \cdot 69$ & $37 \cdot 4$ \\
\hline
\end{tabular}

*Calculated from McCance and Widdowson. The composition of foods, 1978. 
$10 \mathrm{~min}$. Free amino acids in the supernatant were estimated using an LKB 4151 Alpha plus amino acid analyser (LKB Instruments Ltd, South Croydon, UK). Urea and ammonia were measured using Sigma diagnostic kit no 640) (Sigma Chemical Co Ltd, Dorset, England). Total proteolytic activity was assayed by the method of Brock et al ${ }^{13}$ using $3 \%$ azocasein $(\mathrm{w} / \mathrm{v})$ in phosphate buffer $(\mathrm{pH} 7 \cdot 0)$ as substrate and incubation period for one hour. Amylase activity was assayed by the method of Dahlquist. $^{14}$

All subjects gave informed, written consent to the protocol approved by the Ethical Committee of the Dunn Nutrition Unit.

Statistical analysis was by analysis of variance using the SPP package for microcomputers, designed by Patrick Royston, London School of Hygiene and Tropical Medicine.

\section{Results}

The wet and dry weights of ileostomy effluent on the various diets are shown in Table 3 , and all are within the range expected in healthy ileostomists. ${ }^{15}$ Total effluent excretion on LND was 329 (range 226-481) $\mathrm{g} /$ day, and excretion of solids $26 \cdot 0$ (range $21 \cdot 4-32 \cdot 1$ ) $\mathrm{g} /$ day. Total excretion was significantly greater on high fibre diet (LND $v$ HFD $\mathrm{t}=8 \cdot 6, \mathrm{p}<0 \cdot 001$ ) but not on soya bean diets; effluent dry weight was greater on both soya bean diets (LND $v$ WSB $\mathrm{t}=7 \cdot 2, \mathrm{p}<0 \cdot 001$;

Table 3 Ileostomy outputs

\begin{tabular}{|c|c|c|c|c|c|}
\hline $\begin{array}{l}\text { Ileostomy } \\
\text { output } \\
(\mathrm{g} / 24 \mathrm{~h})\end{array}$ & & Diet I & Diet II & Diet III & Diet IV \\
\hline $\begin{array}{l}\text { Wet } \\
\text { weight }\end{array}$ & $\begin{array}{l}\text { Mean (SE) } \\
\text { Range }\end{array}$ & $\begin{array}{l}329^{: *}(45) \\
226-481\end{array}$ & $\begin{array}{l}490^{a}(44) \\
337-671\end{array}$ & $\begin{array}{l}465^{\mathrm{a}}(34) \\
345-586\end{array}$ & $\begin{array}{l}7\left(12^{\mathrm{b}}(29)\right. \\
574-777\end{array}$ \\
\hline $\begin{array}{l}\text { Dry } \\
\text { weight }\end{array}$ & $\begin{array}{l}\text { Mean (SE) } \\
\text { Range }\end{array}$ & $\begin{array}{l}26 \cdot\left(0^{11}(1 \cdot 6)\right. \\
21 \cdot 4-32 \cdot 1\end{array}$ & $\left\{\begin{array}{l}56 \cdot 5^{\text {b }}(5 \cdot 9) \\
31 \cdot 3-70 \cdot 7\end{array}\right.$ & $\begin{array}{l}49 \cdot 1^{\mathrm{h}}(3 \cdot 2) \\
39 \cdot 4-60 \cdot 3\end{array}$ & $\left\{\begin{array}{l}71 \cdot 3^{c}(2 \cdot 1) \\
66 \cdot 4-80 \cdot 3\end{array}\right.$ \\
\hline
\end{tabular}

${ }^{*}$ Means in any row with different superscript letters are statistically significantly different.
LND $v$ PSB $\mathrm{t}=7 \cdot 8, \mathrm{p}<0 \cdot 001)$ and high fibre diet (LND $v$ HFD $\mathrm{t}=18 \cdot 8, \mathrm{p}<0 \cdot 001)$. There was no difference in total effluent excretion or dry weights between the two soya bean diets, but the high fibre diet was greater than both. (Total effluent: WSB $v$ HFD $\mathrm{t}=4 \cdot 0, \mathrm{p}<0 \cdot 025 ;$ PSB $v$ HFD $\mathrm{t}=5 \cdot 4, \mathrm{p}<0 \cdot 005$. Dry weight: WSB $v$ HFD $\mathrm{t}=3.4, \mathrm{p}<0.025$; PSB $v$ HFD $t=5 \cdot 8, p<0 \cdot(005)$. The wet and dry weights of effluent were both significantly related to the amount of dietary fibre intake (total effluent $r=0.88$, $\mathrm{p}<0 \cdot 001$; solids $\mathrm{r}=(0 \cdot 85, \mathrm{p}<0 \cdot 001)$.

Total nitrogen excretion (Table 4) in ileostomy effluent on the low $\mathrm{N}$ diet was $0.91(0 \cdot 04) \mathrm{g} /$ day (mean (SE)), range $0.82-1.09 \mathrm{~g} /$ day and this has been taken as arising entirely from endogenous nitrogen secreted into the gut, since the small amount of dietary nitrogen $(0 \cdot 17 \mathrm{~g})$ would probably have been absorbed. Despite the low protein intake, exocrine pancreatic function was normal ${ }^{14}$ on the low $\mathrm{N}$ diet; effluent amylase $223(48)$ units $/ \mathrm{ml}$; total proteolytic activity $4.6 \times 10^{4}(0.7) \mu \mathrm{g}$ azocasein hydrolysed $/ \mathrm{h} / \mathrm{ml}$. When whole soya beans were added to low $\mathrm{N}$ diet, nitrogen losses increased to $2 \cdot 26(0 \cdot 15)$ g/day (range $1.66-2.69 \mathrm{~g} /$ day), but were significantly lower when the same amount of beans was well ground and fed (pureed beans) $\mathrm{N}=1.42(0.12) \mathrm{g} /$ day; range $1.03-$ $1.83 \mathrm{~g} /$ day $(\mathrm{t}=8 \cdot 0, \mathrm{p}<0 \cdot 001)$. Nitrogen digestibility $(\%)$ (Table 4$)$ on the pureed bean diet $(90 \cdot 1(1 \cdot 3))$ was significantly higher than with whole beans $(73 \cdot 0$ $(3 \cdot 3))$. Nitrogen loss on the high fibre diet was $2 \cdot 17$ $(0 \cdot 11) \mathrm{g} /$ day, range $1 \cdot 80-2 \cdot 35 \mathrm{~g} /$ day and digestibility was similar to pureed bean diet $(88 \cdot 5(0 \cdot 7))$.

Eighty to $85 \%$ of effluent $\mathrm{N}$ was in the insoluble (pellet) fraction except on the low $\mathrm{N}$ diet where it was $66 \%$ (Table 4 ).

COMPOSITION OF ILEOSTOMY NITROGEN LOSS Table 5 shows the composition of ileostomy effluent $\mathrm{N}$ on the various diets. The protein content of the homogenate mirrored total $\mathrm{N}$ loss and ranged from $2.69 \mathrm{~g} /$ day on low $\mathrm{N}$ diet to $7.45 \mathrm{~g} /$ day on whole soya bean diet. Protein loss on whole soya beans was significantly higher than pureed beans $(t=5 \cdot 5$,

Table 4 Effect of diet on ileostomy nitrogen losses (g/day), mean (SE)

\begin{tabular}{|c|c|c|c|c|}
\hline & Low nitrogen diet ( $L N D)$ & $\begin{array}{l}\text { LND+whole soya beans } \\
(L N D+W S B)\end{array}$ & $\begin{array}{l}\text { L.ND+ pureed soya beans } \\
(L . N D+P S B)\end{array}$ & $\begin{array}{l}\text { High fibre } \\
\text { diet }(H F D)\end{array}$ \\
\hline Total N & $\left(0.91^{\mathrm{a}}(0 \cdot() 4)\right.$ & $2 \cdot 26^{\mathrm{b}}(0 \cdot 1.5)$ & $1 \cdot 42^{\mathrm{c}}(0 \cdot 12)$ & $2 \cdot 17^{b}(0 \cdot 11)$ \\
\hline$\% \mathrm{~N}$ absorbedt & & $73 \cdot 0 \cdot(3 \cdot 3)$ & 9()$\cdot 1^{b}(1 \cdot 3)$ & $88.5^{\mathrm{b}}(0.7)$ \\
\hline Insoluble $\mathrm{N}$ & $\left(0 \cdot 60^{a}(0 \cdot() 4)\right.$ & $1.92^{\mathrm{b}}(0 \cdot 12)$ & $1 \cdot 13^{c}(0 \cdot 13)$ & $1 \cdot 74^{\mathrm{b}}(0 \cdot 10)$ \\
\hline Soluble N & $\left(0.31^{\circ 1}(0 \cdot(04)\right.$ & $\left(0 \cdot 34^{\circ \prime}(0 \cdot 08)\right.$ & $0.3\left(0^{\mathrm{a}}(0 \cdot(13)\right.$ & $0 \cdot 43^{\mathrm{a}}(0 \cdot(04)$ \\
\hline
\end{tabular}

* Means in any row with different superscript letters are statistically significantly different.

$\frac{\dagger \text { (ileostomy } \mathrm{N} \text { on diet-ileostomy } \mathrm{N} \text { on low } \mathrm{N} \text { diet ) }}{\text { dietary } \mathrm{N} \text {-dietary } \mathrm{N} \text { on low } \mathrm{N} \text { diet }} \times 100$ 
Table 5 Composition of small bowel nitrogen losses, mean (SE)

\begin{tabular}{|c|c|c|c|c|}
\hline & $l . N I)$ & $L N D+W S B$ & $L N D+P S B$ & $H F I$ \\
\hline $\operatorname{Protcin}(\mathrm{g} / \mathrm{day})$ & $2 \cdot 69^{a *}(0 \cdot 11)$ & $7 \cdot 45^{\mathrm{h}}(0 \cdot 48)$ & $4 \cdot 19^{\circ}(0 \cdot 21)$ & $6.15^{\mathrm{h}}(0.29)$ \\
\hline Free amino acidt conc $(\mu \mathrm{mol} / \mathrm{ml})$ & $6 \cdot 9\left(0^{\prime \prime}(0 \cdot 65)\right.$ & $20 \cdot 48^{\mathrm{h}}(2 \cdot 42)$ & $14 \cdot 50)^{\mathrm{ath}}(1 \cdot 68)$ & $12 \cdot 17^{\cdot 1}(2 \cdot 37)$ \\
\hline Free amino acidt output ( $\mu \mathrm{mol})$ & $2561^{: 1}(201)$ & $11410^{\mathrm{h}}(2813)$ & $7177^{\mathrm{h}}(281.3)$ & $8.364^{\mathrm{h}}(18.3 .3)$ \\
\hline Urea $(\mathrm{mmol} / \mathrm{l})$ & $3 \cdot() 1^{3}(0 \cdot 17)$ & $3.41^{a}(0.94)$ & $3 \cdot 19^{a}(1 \cdot 87)$ & $4 \cdot(17 \cdot 1 \cdot(1 \cdot 22)$ \\
\hline Ammonia (mmol/l) & $1 \cdot 89 \cdot(0 \cdot 31)$ & $1 \cdot 41^{13}(0 \cdot 6(0)$ & $2 \cdot 56^{\mathrm{a}}(() \cdot 92)$ & $2 \cdot 65^{\prime \prime}((1) \cdot 84)$ \\
\hline
\end{tabular}

*Means in any row with different superscript letters are statistically significantly different. $\mathrm{n}=3$.

$\mathrm{p}<0 \cdot 005$ ). Urea losses were low and ranged from 3.01-4.07 mmol/l, whilst ammonia losses were similarly low at $1.41-2.65 \mathrm{mmol} / \mathrm{l}$.

Total free amino acid concentrations and outputs on the different diets for three subjects are shown in Table 5 and Table 6 shows the concentrations and outputs of the individual amino acids. On LND, the amino acids with the highest concentrations are glycine, proline, alanine, valine and glutamic acid; and those with lowest concentration are D-Lallohydroxylysine, citrulline and methionine. Bearing in mind the statistical limitations of data for only three patients, analysis of variance for free amino acid concentrations between soya bean diets and high fibre diets showed significant differences in the following: arginine, beta-aminoisobutyric acid $(p<0.001)$; leucine, phenylalanine, methionine, histidine $(\mathrm{p}<0.005)$; alpha-aminobutyric acid, gamma-aminobutyric acid $(p<0 \cdot 01)$, glutamine, proline, alanine, valine, and isoleucine $(\mathrm{p}<0 \cdot 05)$.

In Table 7 the contribution of the various fractions towards small bowel $\mathrm{N}$ loss is shown. Proteins account for $45-51 \%$, amino acids for $6-11 \%$ and urea and ammonia for approximately $5 \%$ of ileal $\mathrm{N}$ losses on the various diets.

Each subject provided a sample directly from the stoma for bacterial counts. Total aerobes were $5.5 \times 10^{7}(1 \cdot 1)$, range $4.8 \times 10^{5}-8.2 \times 10^{\times}$and total

Table 6 Free amino acid concentrations ( $\mu \mathrm{mol} / \mathrm{ml})$ and outputs ( $\mu \mathrm{mols})$ in ileal effluent, mean (SE)

\begin{tabular}{|c|c|c|c|c|c|c|c|c|}
\hline & \multicolumn{2}{|l|}{$L N D(n=6)$} & \multicolumn{2}{|c|}{$L N D+W S B(n=3)$} & \multicolumn{2}{|c|}{$L N D+P S B(n=3)$} & \multicolumn{2}{|l|}{$H F D(n=6)$} \\
\hline & Conc & Output & Conc & Output & Conc & Output & Conc & Output \\
\hline Aspartic acid & $0.24(0.5)$ & $103 \cdot 2(27 \cdot 2)$ & $0.55(0.07)$ & $307 \cdot 9(77 \cdot 3)$ & $0.43(0.25)$ & $230 \cdot 7(148 \cdot 2)$ & $0 \cdot 66(0 \cdot 23)$ & $456 \cdot 6(169 \cdot 4)$ \\
\hline Threonine & $0 \cdot 18(0 \cdot(03)$ & $72 \cdot 3(15 \cdot 8)$ & $0.70(0 \cdot 15)$ & $397 \cdot 4(130.9)$ & $0.48(0 \cdot 11)$ & $243.6(82.9)$ & $0.32(0 \cdot 10)$ & $228 \cdot 0(73 \cdot 6)$ \\
\hline Serine & $0 \cdot 14(0 \cdot(03)$ & $58 \cdot 1(17 \cdot 2)$ & $0.57(0.17)$ & $330 \cdot 5(138 \cdot 7)$ & $0.35(0.14)$ & $185 \cdot 0(89.8)$ & $0.19(0.09)$ & $133 \cdot 1(66 \cdot 6)$ \\
\hline Asparagine & $0 \cdot 11(0 \cdot(05)$ & $53 \cdot 5(26 \cdot 5)$ & $0.45(0 \cdot 32)$ & $288 \cdot 1(219 \cdot 2)$ & $0.27(0.27)$ & $157 \cdot 3(157 \cdot 3)$ & $0 \cdot 25(0 \cdot 16)$ & $181 \cdot 2(114 \cdot 7)$ \\
\hline Glutamine & $0.33(0 \cdot(08)$ & $142 \cdot 2(39 \cdot 7)$ & $2 \cdot 42(0 \cdot 46)$ & $1372(435 \cdot 5)$ & $0.81(0.41)$ & $424 \cdot 7(232 \cdot 4)$ & $0.70(0 \cdot 39)$ & $509 \cdot 1(281 \cdot(0)$ \\
\hline Proline & $0.62(0.15)$ & $227.4(38.4)$ & $1 \cdot 36(0 \cdot(03)$ & $734(8.5 \cdot 1)$ & $1 \cdot 11(0 \cdot 21)$ & $537 \cdot 2(103 \cdot 7)$ & $0.62(0.17)$ & $438 \cdot 2(125 \cdot 2)$ \\
\hline Glycine & $1 \cdot 36(0 \cdot 31)$ & $459 \cdot 6(57 \cdot 9)$ & $1 \cdot 75(0 \cdot 31)$ & $921 \cdot 9(117 \cdot 6)$ & $1 \cdot 88(0.33)$ & $916 \cdot 5(174 \cdot 7)$ & $1.44(0 \cdot 20)$ & $970 \cdot 7(137 \cdot 8)$ \\
\hline Alanine & $0.48(0.08)$ & $170 \cdot 5(9 \cdot 3)$ & $1 \cdot 43(0 \cdot 13)$ & $783 \cdot 7(156 \cdot 1)$ & $1 \cdot 11(0 \cdot 16)$ & $532 \cdot 1(68 \cdot())$ & $0.79(0.12)$ & $538 \cdot 2(94 \cdot 2)$ \\
\hline Valine & $0 \cdot 38(0 \cdot(06)$ & $136 \cdot 2(14 \cdot())$ & $1 \cdot 02(0 \cdot 18)$ & $577 \cdot 1(170 \cdot 2)$ & $0 \cdot 80(0 \cdot 06)$ & $389 \cdot 5(52 \cdot 6)$ & $0.47(0.09)$ & $326 \cdot 0(71 \cdot 3)$ \\
\hline Cystine & $0 \cdot 08(0 \cdot(02)$ & $28 \cdot 6(6 \cdot 9)$ & $0 \cdot 15(0 \cdot 01)$ & $82 \cdot 8(12 \cdot 7)$ & $0 \cdot 23(0 \cdot(07)$ & $110 \cdot 6(27 \cdot 5)$ & $0 \cdot 12(0 \cdot 02)$ & $84 \cdot 4(18 \cdot 3)$ \\
\hline Methionine & $0 \cdot 06(0 \cdot(01)$ & $24 \cdot 1(4 \cdot 4)$ & $0 \cdot 20(0 \cdot(03)$ & $107 \cdot 0(17 \cdot 7)$ & $0 \cdot 16(0 \cdot(02)$ & $79 \cdot 8(16 \cdot())$ & $0.09(0.01)$ & $59.8(7.9)$ \\
\hline Isoleucine & $0 \cdot 24(0 \cdot() 2)$ & $93.7(12.6)$ & $0 \cdot 83(0 \cdot 17)$ & $473 \cdot 3(153 \cdot 3)$ & $0.57(0 \cdot 05)$ & $283 \cdot 6(56 \cdot 6)$ & $0 \cdot 39(0 \cdot 08)$ & $271 \cdot 8(59 \cdot 8)$ \\
\hline Tyrosine & $(0 \cdot 21(() \cdot() 3)$ & $80 \cdot 5(16 \cdot 0)$ & $0.63(0 \cdot 12)$ & $355 \cdot 4(111 \cdot 5)$ & $0.47(0 \cdot 06)$ & $235 \cdot 9(56 \cdot 7)$ & $0.33(0 \cdot(07)$ & $229.9(53.6)$ \\
\hline Phenylalanine & $0 \cdot 21(() \cdot(12)$ & $80 \cdot 2(11 \cdot 5)$ & $0.75(0 \cdot(09)$ & $416 \cdot 7(103 \cdot 4)$ & $0.57((0 \cdot() 6)$ & $283 \cdot 2(70) \cdot(0)$ & $0 \cdot 24(0 \cdot 05)$ & $166 \cdot 7(41 \cdot 0)$ \\
\hline $\begin{array}{l}\text { D.L. Allo- } \\
\text { hydroxylysine }\end{array}$ & $0 \cdot 02(0 \cdot 01)$ & $6 \cdot 5(2 \cdot 9)$ & $0 \cdot 01(0 \cdot() 1)$ & $5 \cdot 3(3 \cdot 6)$ & $0 \cdot 04(0 \cdot 02)$ & $17 \cdot 6(9 \cdot 1)$ & $0.02(0 \cdot 01)$ & $15 \cdot 3(5 \cdot 5)$ \\
\hline Lysine & $0 \cdot 15(0 \cdot(04)$ & $55 \cdot 1(16 \cdot 2)$ & $0.61(0.61)$ & $408 \cdot 3(408 \cdot 4)$ & $0.37(0.29)$ & $209 \cdot 6(172 \cdot 5)$ & $(0 \cdot 48(0 \cdot 24)$ & $337 \cdot 3(172.9)$ \\
\hline Arginine & $0 \cdot 28(0 \cdot(04)$ & $108 \cdot 0(17 \cdot 7)$ & $1 \cdot 81(0 \cdot 30)$ & $1019 \cdot 0(296 \cdot 5)$ & $0.71(0.08)$ & $354 \cdot()(79 \cdot 8)$ & $0.45(0 \cdot 12)$ & $316 \cdot 1(90 \cdot 5)$ \\
\hline Histidine & $0.21(0.03)$ & $75 \cdot 3(9 \cdot 6)$ & $0 \cdot 64(0.03)$ & $347 \cdot 2(56 \cdot 9)$ & $0.44(0 \cdot 01)$ & $210 \cdot 9(16 \cdot 2)$ & $0 \cdot 33(0 \cdot 05)$ & $222 \cdot 8(36 \cdot 6)$ \\
\hline 1-Methyl-histidine & $0 \cdot 36(0 \cdot 13)$ & $128 \cdot 2(52 \cdot 6)$ & $0.89(0.46)$ & $426 \cdot 3(227 \cdot 3)$ & $0.55(0.28)$ & $238 \cdot 2(121 \cdot 2)$ & $0.42(0: 16)$ & $282 \cdot 6(110 \cdot 6)$ \\
\hline 3-Methyl-histidine & $0 \cdot 19(0 \cdot(12)$ & $75 \cdot()(11 \cdot 0)$ & $0.47(0.13)$ & $270 \cdot 2(106 \cdot 4)$ & $0.21(0 \cdot 12)$ & $109.5(68.4)$ & $0 \cdot 13(0 \cdot 08)$ & $90 \cdot 5(57 \cdot 3)$ \\
\hline Citrulline & $0 \cdot(01(0 \cdot(01)$ & $3 \cdot 2(2 \cdot 3)$ & $0 \cdot 05(0 \cdot 03)$ & $21 \cdot 4(21 \cdot 4)$ & $0 \cdot 10(() \cdot(04)$ & $41 \cdot 6(25 \cdot 1)$ & $0 \cdot 03(0 \cdot(02)$ & $18 \cdot 3(10 \cdot 6)$ \\
\hline Ornithine & $(0 \cdot 08(0 \cdot(02)$ & $24 \cdot 8(4 \cdot 5)$ & $0 \cdot 10(0 \cdot(05)$ & $61 \cdot 1(38 \cdot 4)$ & $0 \cdot 08(0 \cdot(03)$ & $38 \cdot 3(9 \cdot 1)$ & $0 \cdot 06(0 \cdot 01)$ & $40 \cdot 0(5 \cdot 5)$ \\
\hline$\alpha$-Amino butyric acid & $0 \cdot 08(0 \cdot(03)$ & $24 \cdot 2(5 \cdot 5)$ & $0 \cdot 23(0 \cdot(1) 2)$ & $123 \cdot 8(12 \cdot 3)$ & $0 \cdot 30(0 \cdot() 8)$ & $142 \cdot 0(28 \cdot 6)$ & $(0 \cdot 11(0 \cdot(01)$ & $70 \cdot 7(7 \cdot 9)$ \\
\hline $\begin{array}{l}\beta \text {-Amino isobutyric } \\
\text { acid }\end{array}$ & $0 \cdot 11(0 \cdot 05)$ & $45 \cdot 9(23 \cdot(0)$ & $0 \cdot 00$ & $0 \cdot 00$ & $0 \cdot 00$ & $0 \cdot 00$ & $1 \cdot 52(0 \cdot 25)$ & $999 \cdot 0(143 \cdot 2)$ \\
\hline$\gamma$-Amino butyric acid & $(0 \cdot(17(0) \cdot(04)$ & $17 \cdot 8(9 \cdot 2)$ & $0 \cdot 02(0 \cdot(02)$ & $12 \cdot 4(12 \cdot 4)$ & $0 \cdot 03(0 \cdot() 3)$ & $16 \cdot 2(16 \cdot 2)$ & $0 \cdot 14(0 \cdot(02)$ & $93 \cdot 3(8 \cdot 8)$ \\
\hline
\end{tabular}


Table 7 Composition of Nlosses on each diet (g/day), mean (SE)

\begin{tabular}{|c|c|c|c|c|}
\hline & $L N D$ & $L N D+W S B$ & $L N D+P S B$ & $H F D$ \\
\hline Total N & $0.91^{4 *}(0.04)$ & $2 \cdot 26^{\prime \prime}(0 \cdot 15)$ & $1.42^{c}(0 \cdot 12)$ & $2 \cdot 17^{\mathrm{b}}(0 \cdot 17)$ \\
\hline Protein N & $0.43^{\mathrm{a}}(0.03)(47 \%)$ & $1 \cdot 16^{\mathrm{h}}(0 \cdot 13)(51 \%)$ & $0.68^{\circ}(0.06)(48 \%)$ & $0.99^{\mathrm{h}}(0.08)(45 \%)$ \\
\hline Free amino acid $\mathrm{N}$ & $0 \cdot 05^{\mathrm{a}}(0 \cdot 001)$ & $0 \cdot 26^{\mathrm{b}}(0 \cdot(0) 2)$ & $0 \cdot 16^{b}(0 \cdot 001)$ & $0 \cdot 20^{\mathrm{h}}(0 \cdot 001)$ \\
\hline Urea $\mathrm{N}$ & $0 \cdot\left(04^{\prime \prime}(0 \cdot 01)\right.$ & $\left(0 \cdot(1) 6^{1}(0) \cdot(12)\right.$ & $0.05^{\prime \prime}(0 \cdot 03)$ & $0.08^{\mathrm{a}}(0 \cdot 05)$ \\
\hline Ammonia $\mathrm{N}$ & $0 \cdot 01^{\prime \prime}(0 \cdot 0(0) 1)$ & $\left(0 \cdot\left(01^{a}(0) \cdot 005\right)\right.$ & $0 \cdot\left(02^{\mathrm{a}}(0 \cdot 004)\right.$ & $0 \cdot 03^{\mathrm{a}}(0 \cdot 004)$ \\
\hline
\end{tabular}

${ }^{*}$ Means in any row with different letters are statistically significantly different.

anaerobes $4.2 \times 10^{6}(0 \cdot 72)$, range $8.0 \times 10^{5}-2 \cdot 6 \times 10^{7}$ which were within the range seen in other ileostomists. ${ }^{16}{ }^{17}$ Average breath hydrogen was 4 ppm and in no subject rose above $7 \mathrm{ppm}$.

\section{Discussion}

Small intestinal nutrient absorption in man has been studied by a variety of techniques including intestinal perfusion and using patients with terminal ileostomies. Intestinal intubation delays gastric emptying and shortens small intestinal transit time,$^{18}$ which in turn influences nutrient absorption. ${ }^{14}$ So we have used healthy ileostomists to measure endogenous nitrogen losses and evaluate the effect of dietary factors on nitrogen losses from the small intestine. Healthy ileostomists have a mouth to caecum transit time identical to normal subjects. ${ }^{1 \times}$ Though microbial counts are higher in ileostomy effluent as compared with ileal fluid from normal subjects, there is little bacterial fermentation as breath hydrogen is always low, does not rise after meals, and short chain fatty acid concentrations are also very low. ${ }^{211}$ In other respects, the digestive function of ileostomists resembles that of the normal gut in that ileal effluent contains substantial amounts of pancreatic enzymes $^{22.33}$ and ileal excretion of fat, protein and carbohydrate from mixed diets is no greater than faecal excretion in normal healthy subjects. ${ }^{19+4}$

Although there are numerous reports of total nitrogen losses from patients with a terminal ileostomy, ${ }^{3-5}$ values range from $0 \cdot 6-2 \cdot 4 \mathrm{~g} / \mathrm{day}$, little is known of the relative contributions from diet and endogenous sources. We measured endogenous nitrogen losses from distal small bowel in healthy ileostomists by feeding them an essentially nitrogen free diet $(0.17 \mathrm{~g} \mathrm{~N} /$ day $)$. Total nitrogen loss in the ileal effluent was $0.91 \mathrm{~g} /$ day. Most of the endogenous nitrogen is contributed by secretions, mucus, and shed epithelial cells. As dietary proteins are known to stimulate pancreatic exocrine secretion ${ }^{\text {?s }}$ the low nitrogen diet may not have adequately stimulated the pancreas. Hence, there would be an underestimation of endogenous nitrogen loss measured when the patients ate such a diet. So, total proteolytic activity (TPA) and amylase activity in the ileal effluent were measured. Total proteolytic activity $\left(4.64 \times 10^{4} \mu \mathrm{g}\right.$ azocasein hydrolysed/h/ml) and amylase activity $(222.7 \mathrm{U} / \mathrm{ml})$ on the low nitrogen diet were within the range seen in healthy ileostomists ${ }^{2-4}$ indicating that there was adequate stimulation of pancreatic exocrine activity.

The importance of physical form of food on rate of digestion of carbohydrates in cereals and legumes has been shown..$^{27}$ We evaluated the effect of physical form (whole and pureed) of soya beans on nitrogen losses. Nitrogen losses with whole beans $(2 \cdot 3 \mathrm{~g} / \mathrm{day})$ is significantly higher than pureed beans $(1.4 \mathrm{~g} / \mathrm{day})$ and percentage absorption of nitrogen from whole beans $(73 \%)$ is significantly lower than pureed beans $(90 \%)$. The probable reason for this difference in digestibility is the greatly increased surface area when the beans are pureed. This provides easy access to digestive enzymes and hence more rapid and complete digestion and absorption. The effect of physical form of food in determining nitrogen losses from the distal small intestine may be important because beans, which form a significant part of dietary protein in developing countries, are usually eaten whole.

Previous studies show that increase in intake of non-starch polysaccharides (NSP) is associated with increase in faecal nitrogen losses. ${ }^{2 \times}{ }^{29}$ The increase in faecal nitrogen is mainly bacterial ${ }^{311}$ and may be related to increased bacterial growth on high NSP (dietary fibre) diets. Do small intestinal events contribute to increased nitrogen losses? It has been shown by in vitro studies ${ }^{31}$ that NSP interferes with pancreatic enzyme activity. , Non-starch polysaccharides also increases the volume and weight of intestinal contents, may act as a physical barrier impairing digestion and absorption and cause morphological changes in the structure of the small bowel. ${ }^{32.33}$ To gauge the effect of NSP on ileal nitrogen losses, we fed the patients a high NSP diet and compared the ileal nitrogen losses with those seen with the soya bean diets, containing only half the NSP, and with a larger group of free living ileo- 
stomists consuming standard UK diets containing about $15 \mathrm{~g} \mathrm{NSP/day} \mathrm{whom} \mathrm{we} \mathrm{had} \mathrm{previously}$ studied. ${ }^{15}$ Ileal nitrogen losses in the free living ileostomists $(2 \cdot 1 \mathrm{~g} / \mathrm{day})$ is similar to the patients fed high NSP diet $(2 \cdot 2 \mathrm{~g} /$ day) and percentage absorption of nitrogen on high NSP diet $(88.5 \%)$ is similar to pureed bean diet $(90 \cdot 1 \%)$. Similarly $\mathrm{N}$ losses on the HFD are no greater than with whole soya beans, although NSP intakes are more than double. These results suggest that dietary fibre as consumed in a normal high fibre mixed diet, plays a small role, if any in influencing small bowel nitrogen losses.

The exact forms of nitrogen entering the large bowel are largely unknown. Macfarlane et al" have shown that the solubility of proteins is an important factor determining the rate of their fermentation. By low speed centrifugation we separated ileostomy effluent into soluble and insoluble fractions. Insoluble fraction accounts for $66 \%$ of endogenous $\mathrm{N}$ losses and $80-85 \%$ of nitrogen lost on other diets. Protein accounted for the major portion of effluent nitrogen on all diets and ranged from $45 \%$ of total $N$ on high fibre diet to $51 \%$ on the whole soya bean diet. Only small amounts of urea and ammonia were found, accounting for less than $5 \%$ of total $\mathrm{N}$. Urea $^{3435}$ and creatinine ${ }^{36}$ concentrations in distal ileal fluid are usually similar to blood levels. Very little (about $100 \mathrm{mg}$ ) uric acid is reported to enter the large bowel each day ${ }^{37}$ and only small amounts of amino acids are found in ileal fluids ${ }^{36}{ }^{38}$ In the present study amino acids contribute a small fraction to ileal nitrogen loss ranging from $6.0 \%$ on low nitrogen diet to $11 \%$ on the pureed bean diet. High concentrations of beta-amino isobutyric acid are seen on high fibre diet $(1.5 \mu \mathrm{mol} / \mathrm{ml})$. Beta-amino isobutyrate is a degradation product of thymine ${ }^{39}$ so the increased levels may be due either to increased small intestinal cell turnover, known to occur with high fibre diets, or be derived from plant cell nucleic acids. By difference, most of the unaccounted for nitrogen must be in the form of peptides (30-40\%). This suggests that proteins and peptides are the major forms of nitrogen lost from the small bowel ( $80-85 \%)$.

In conclusion, this study suggests that obligatory $\mathrm{N}$ losses from the small intestine are about $1.0 \mathrm{~g} /$ day, and nitrogen loss is mainly in the form of proteins and peptides. Physical form of food influences $\mathrm{N}$ loss but not dietary fibre content.

The authors wish to record their thanks to Geoff Hudson, Peter John, and Brian Baker for help with the nitrogen and amino acid analyses, to Sheila Bingham for designing and preparing the diets, and to the six ileostomy volunteers who were such cheerful and reliable subjects. AC was in receipt of a grant from the Wellcome Trust.

\section{References}

1 Cummings JH. Fermentation in the human large intestine: evidence and implications for health. Lancet 1983 ; i: 1206-9.

2 Macfarlane GT, Cummings JH, Allison C. Protein degradation by human intestinal bacteria. $J$ Gen Microbiol 1986; 132: 1647-56.

3 Smiddy FG, Gregory SD, Smith IB, Goligher JC. Fecal loss of fluid, electrolytes and nitrogen in colitis before and after ileostomy. Lancet 1960; i: 14-9.

4 Nuguid TP, Bacon HE, Boutwell J. An investigation of the volume of output and chemical content of ileal discharges following total colectomy and ilcostomy. Surg Gynecol Obstet 1961; 113: 733-42.

5 Kramer $P$. The effect of varying sodium loads on the ileal excreta of human ileostomised subjects. $J$ Clin Invest 1966; 45: 1710-8.

6 Gibson JA, Sladen GE, Dawson AM. Protein absorption and ammonia production: the effects of dietary protein and removal of the colon. Br J Nutr 1976; 35: 61-5.

7 Sandberg AS, Anderson H, Hallgren B, Hasselblad K, Isaksson B, Hulten L. Experimental model for in vivo determination of dietary fibre and its effect on the absorption of nutrients in the small intestine. Br J Nutr 1981; 45: 283-94.

8 Cummings JH, Hill MJ, Bone ES, Branch WJ, Jenkins DJA. The effect of meat protein and dietary fibre on colonic function and metabolism. II. Bacterial metabolites in feces and urine. Am J Clin Nutr 1979; 32: 2094-101.

9 Macfarlane GT, Allison C. Utilisation of protein by human gut bacteria. FEMS Microbiol Ecol 1986; 38: 19-24.

10 Gibson JA, Park NJ, Sladen GE, Dawson AM. The role of the colon in urea metabolism in man. Clin Sci Mol Med 1976; 50: 51-9.

11 Mitchell $\mathrm{HH}$. The nutrients contained in foods. I. The analysis of food materials. In: Comparative nutrition of man and domestic animals. Vol. 2. New York and London: Academic Press, 1964: 313-4.

12 Lowry $\mathrm{OH}$, Roseborough NJ, Farr AJ, Randall RJ. Protein measurement with the folin phenol reagent. J Biol Chem 1951; 143: 265-75.

13 Brock FM, Forsberg CW, Buchanan-Smith JG. Proteolytic activity of rumen micro-organisms and effects of proteinase inhibitors. Appl Environ Microbiol 1982; 44: 561-9.

14 Dahlquist A. A method for determination of amylase in intestinal content. Scand J Clin Lab Invest 1962; 14: $145-51$.

15 McNeil NI, Bingham S, Cole TJ, Grant AM, Cummings JH. Diet and health of people with an ileostomy. 2. Ileostomy function and nutritional state. Br J Nutr 1982; 47: 407-15.

16 Gorbach SL, Nahas L, Weinstein L. Studies of intestinal microflora. IV. The microflora of ileostomy effluent: a unique microbial ecology. Gastroenterology 1967; 53: 874-80.

17 Finegold SM, Sutter VL, Boyle JD, Shimada K. The normal flora of ileostomy and transverse colostomy effluents. J Infect Dis 1970; 122: 376-81. 
18 Read NW, Al-Janabi MN. Small intestinal intubation delays gastric emptying but accelerates small bowel transit time [Abstract]. Gut 1982; 23: A443.

19 Holgate AM, Read NW. Relationship between small bowel transit time and absorption of a solid meal. Influence of metoclopramide, magnesium sulphate and lactulose. Dig Dis Sci 1983; 28: 812-9.

20 Chapman RW, Sillery JK, Graham MM, Saunders DR. Absorption of starch by healthy ileostomates: effect of transit time and of carbohydrate load. Am J Clin Nutr $1985 ; 41: 1244-8$.

21 Englyst HN, Cummings JH. Digestion of the carbohydrates of banana (Musa paradisiaca Sapientum) in the human small intestine. Am J Clin Nutr 1986; 44: 42-50.

22 Bohe M, Borgstrom A, Genell S, Ohlsson K. Determination of immunoreactive trypsin, pancreatic elastase, and chymotrypsin in extracts of human feces and ileostomy drainage. Digestion 1983; 27: 8-15.

23 Goldberg DM, McAllister RA, Roy AD. Studies on human intestinal proteolysis. Scand J Gastroenterol 1968; 13: 193-201.

24 Hill GL. Ileostomy: surgery, physiology and management. New York: Grune \& Stratton, 1976.

25 Roy AD, Campbell R, Goldberg DM. Effect of diet on the trypsin and chymotrypsin output in the stools of patients with an ileostomy. Gastroenterology 1967; 53: 584-9.

26 Snow P, O'Dea K. Factors affecting the rate of starch hydrolysis in food. Am J Clin Nutr 1981; 34: 2721-7.

27 Wong S, O'Dea K. Importance of physical form rather than viscosity in determining the rate of starch hydrolysis in legumes. Am J Clin Nutr 1983; 37: 66-70.

28 Southgate DAT, Durnin JVGA. Calorie conversion factors. An experimental reassessment of the factors used in the calculation of the energy value of human diets. Br J Nutr 1970; 24: 517-35.

29 Cummings JH, Southgate DAT, Branch W, et al. The digestion of dietary pectin in the human gut, and its effect on calcium absorption and large bowel function. Br J Nutr 1979; 41: 477-85.

30) Stephen AM, Cummings JH. The influence of dietary fibre on faecal nitrogen excretion in man [Abstract]. Proc Nutr Soc 1979; 38: 141 A.

31 Dunaif $\mathrm{G}$, Schneeman BO. The effect of dietary fibre on human pancreatic enzyme activity in vitro. $A m J$ Clin Nutr 1981; 34: 1034-5.

32 Cassidy MM, Lightfoot FG, Gran LE, Story JA, Kritchevsky D, Vahouny GV. Effect of chronic intake of dietary fibre on the ultrastructural topography of rat jejunum and colon: a scanning electron microscopy study. Am J Clin Nutr 1981; 34: 218-28.

33 Tasman-Jones C, Jones AL, Owery RL. Jejunal morphological consequences of dictary fibre in rats. Gastroenterology 1978; 74: 1102.

34 Gibson JA, Park NJ, Sladen GE, Dawson AM. The role of the colon in urea metabolism in man. Clin Sci Mol Med 1976; 50: 51-9.

35 Cummings JH, Milton-Thompson GJ, Billings JA, Misiewicz JJ. The flow rate and composition of ileal contents during fasting and in response to a liquid meal in man. Gut 1976; 17: 817-8.

36 Padovan W, Owens CWI, Ferguson R. Creatinine and amino acid profiles of ileal and fecal fluids. Clin Sci Mol Med 1975; 49: 27P.

37 Chadwick VS, Jones JD, Debongnic JC, Gaginella T, Phillips SF. Urea, uric acid and creatinine fluxes through the small intestine of man [Abstract]. Gut 1977; 18: A944.

38 Adibi AS, Mercer DW. Protein digestion in human intestine as reflected in luminal, mucosal and plasma amino acid concentrations after meals. J Clin Invest 1973; 52: 1586-94.

39 Metzler DE. Biochemistry: the chemical reactions of living cells. New York: Academic Press, 1977. 